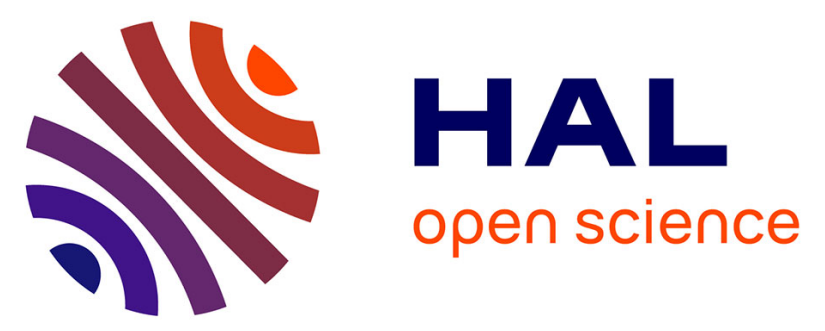

\title{
Experimental evidence of temperature gradients in cavitating microflows seeded with thermosensitive nanoprobes
}

Frédéric Ayela, Manuel Medrano Muñoz, David Amans, Christophe Dujardin, Thomas Brichart, Matteo Martini, Olivier Tillement, Gilles Ledoux

\section{To cite this version:}

Frédéric Ayela, Manuel Medrano Muñoz, David Amans, Christophe Dujardin, Thomas Brichart, et al.. Experimental evidence of temperature gradients in cavitating microflows seeded with thermosensitive nanoprobes. Physical Review E: Statistical, Nonlinear, and Soft Matter Physics, 2013, 88 (4), 10.1103/PhysRevE.88.043016 . hal-01935721

\section{HAL Id: hal-01935721 \\ https://hal.science/hal-01935721}

Submitted on 11 Feb 2021

HAL is a multi-disciplinary open access archive for the deposit and dissemination of scientific research documents, whether they are published or not. The documents may come from teaching and research institutions in France or abroad, or from public or private research centers.
L'archive ouverte pluridisciplinaire HAL, est destinée au dépôt et à la diffusion de documents scientifiques de niveau recherche, publiés ou non, émanant des établissements d'enseignement et de recherche français ou étrangers, des laboratoires publics ou privés. 


\title{
Experimental evidence of temperature gradients in cavitating microflows seeded with thermosensitive nanoprobes
}

\author{
Frédéric Ayela* and Manuel Medrano-Muñoz ${ }^{\dagger}$ \\ Laboratoire des Ecoulements Géophysiques et Industriels (LEGI), UMR5519 UJF Grenoble 1-CNRS, BP 53, 38041 Grenoble Cedex 9, France
}

\begin{abstract}
David Amans, Christophe Dujardin, Thomas Brichart, Matteo Martini, Olivier Tillement, and Gilles Ledoux*
Institut Lumière Matière (ILM), UMR5306 Université Lyon 1-CNRS, Université de Lyon 69622 Villeurbanne Cedex, France
\end{abstract}

(Received 26 April 2013; revised manuscript received 8 July 2013; published 31 October 2013)

\begin{abstract}
Thermosensitive fluorescent nanoparticles seeded in deionized water combined with confocal microscopy enables thermal mapping over three dimensions of the liquid phase flowing through a microchannel interrupted by a microdiaphragm. This experiment reveals the presence of a strong thermal gradient up to $\sim 10^{5} \mathrm{~K} / \mathrm{m}$ only when hydrodynamic cavitation is present. Here hydrodynamic cavitation is the consequence of high shear rates downstream in the diaphragm. This temperature gradient is located in vortical structures associated with eddies in the shear layers. We attribute such overheating to the dissipation involved by the cavitating flow regime. Accordingly, we demonstrate that the microsizes of the device enhance the intensity of the thermal gap.
\end{abstract}

DOI: 10.1103/PhysRevE.88.043016

PACS number(s): 47.55.-t, 64.70.F-, 78.55.Bq, 47.61.-k

While it addresses several societal aspects such as green energy or the catalysis reaction for the elimination of toxic agents, thermodynamic conditions of cavitation are not fully understood. At the macroscale, only wall temperatures have been accessed. Microfluidic systems have proved to be unsurpassed tools in fluid dynamics. From a fundamental point of view, they remain unique features allowing experiments that should be barely attainable at the macroscale. Local pressure drop measurements have demonstrated the validity of the classical laws of hydrodynamics down to a few micrometers [1]. Furthermore, recent studies have shown how microchannels have helped to elucidate the role of aggregates in the properties of nanofluids [2] as well as the influence of nanoparticles in the threshold of cavitation $[3,4]$. Thus, exploring, via nonintrusive methods, the thermal transport of fluids flowing through microchannels is a stimulating challenge.

Optical methods are well suited when nonintrusive measurement techniques are required. The strong temperature dependence of fluorescent materials has been widely studied over the past decades [5-7]. As an illustration, temperature sensors based on the fluorescence decay have been proposed for cryogenic applications [8] and more recently for combined temperature and velocity measurements in liquid and gas flows [9]. Temperature is also known to affect the intensity of the emitted wavelengths of a fluorescent material submitted to a laser irradiation (so-called laser induced fluorescence). Dual tracer fluorescence thermometry has been developed to prevent bias measurements that could be caused by the fluctuation of the illuminating source. This consists of introducing into the fluid a second dye that is independent of the temperature [5]; the ratio of these signals depends only on the temperature. Another approach is to use one single fluorescent material displaying strong differences in the temperature sensitivity of two

\footnotetext{
*Corresponding authors: frederic.ayela@hmg.inpg.fr, gilles.ledoux@univ-lyon1.fr

${ }^{\dagger}$ Present address: Groupe Cryoréfrigérateurs et Cryogénie Spatiale, CEA/DSM/INAC/SBT/GCCS, Grenoble.
}

separated emitting lines [6,7]. In this paper, our study provides three-dimensional- (3D) resolved investigations at the $\mu \mathrm{m}^{3}$ level of the thermal signature of turbulent cavitating flows.

Microflow fields exhibit strong three-dimensional gradients of velocity or temperature. The extrapolation of macroscopic optical methods are thus vulnerable to failure (i) because the thickness of a laser sheet has a significant impact and (ii) because the short diffusion times involved at microscale are incompatible with a continuous illumination. A recent publication addresses the development of two-color laser-induced fluorescence thermometry (LIF) for microfluidic systems [10]. The authors used a pulsed volumetric laser illumination to conduct measurements of a one-dimensional steady-state temperature gradient. Other alternative approaches include tracking individual thermochromic crystal liquid micrometer particles $[11,12]$ but liquid crystals are sensitive to temperature changes only over a limited temperature range. Moreover, temperatures have been measured in laminar microflows by using rhodamine B as a fluorescent dye [13,14] with only average values recorded.

For our experiment we specifically prepared nanohybrid colloids, the synthesis of which has been described elsewhere [15]. Each nanohybrid structure consists of a 5-nm-size gold core coated by a polysiloxane shell of $25 \mathrm{~nm}$ that contains around 400 fluorescein molecules. The nanohybrids are then functionalized by a stabilizing agent [N-(3triethoxysilylpropyl)gluconamide] that ensures the colloidal stability during experiment. The average number of nanoparticles per voxel is, in the current experiment, equal to 5.5, which is below the critical value of 32 particles per voxel (corresponding to a solid volume concentration of $10^{-4}$ ), above which the presence of a solid phase modifies the onset of cavitation [3]. We calibrated the probes by measuring their luminescence spectra under 450-nm excitation wavelength as a function of temperature [from room temperature (RT) up to $600{ }^{\circ} \mathrm{C}$ as dry powder and from RT to $100^{\circ} \mathrm{C}$ in water] using a LINKHAM THMS600P stage. Typical spectra obtained from $20^{\circ}$ to $40^{\circ} \mathrm{C}$ are presented in Fig. 1. By plotting the temperature values as a function of the ratio between the emitted photons 


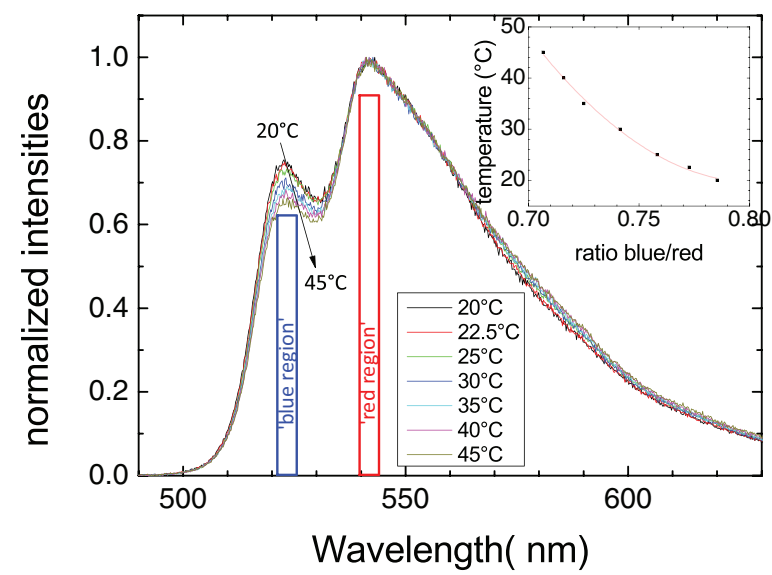

FIG. 1. (Color online) Emission spectra of fluorescent nanoparticles at different temperatures under 485-nm excitation. The ratio between the two peaks is thermally dependent with a relative sensitivity of $\approx 6 \times 10^{-3}{ }^{\circ} \mathrm{C}^{-1}$ at room temperature.

in the "blue region" to the emitted photons in the "red region", we obtained a regular polynomial dependence that we used as the calibration curve for experiments (Fig. 1). The particles' luminescence was also checked as a function of pressure at 8 and 16 bar of nitrogen for temperatures varying from $15{ }^{\circ} \mathrm{C}$ to $80{ }^{\circ} \mathrm{C}$. No effect of the pressure in the range studied was observed. By using the ratio between two parts of the emission spectra, we probe the variation in position of the luminescence. We are therefore insensitive to any variation of intensity of the signal, no matter if is is due to variation of the excitation source or of the collection efficiency. But we are dependent on the wavelength dependence sensitivity of the system. That is why the calibration has been performed on exactly the same setup that we use for the measurement on the microsystems.

We used a custom-made confocal microscope on which microsystems can be easily attached to carry out this experiment. A scheme of the setup is presented in Fig. 2. We analyzed the optical response of the fluorescent particles in the liquid when flowing through the microchannel. We used as an excitation source a doubled femtosecond Ti-sapphire laser working at $82 \mathrm{MHz}$ (TSUNAMI from Spectra-Physics) with output wavelengths between 400 and $500 \mathrm{~nm}$. For the experiment presented herein the laser power was attenuated down to $30 \mu \mathrm{W}$. We focused the laser inside the liquid via a long working distance objective (high-resolution 20× OPTEM from QIOPTIQ) with a numerical aperture of 0.6 . Considering the spatial resolution of system to be $1 \mu \mathrm{m}$ in the $X Y$ plane inside the flow system, and better than $5 \mu \mathrm{m}$ along $Z$ axis, we recorded the average temperature of a an ellipsoidal volume of $1 \times 1 \times 5 \mu \mathrm{m}$ diameters. We then imaged the pin hole (diameter $20-\mu \mathrm{m}$ ) in a monochromator (ANDOR SR500) coupled with an electron magnified CCD (EMCCD Newton from ANDOR). The laser light diffusion is filtered by a long pass edge filter $(488 \mathrm{~nm}$ RazorEdge from SEMROCK). The microsystem is fixed on a double mechanical-piezo stage. The mechanical stage enables the scanning in the $X Y$ directions over $\mathrm{cm}$ distances (100-nm resolution by steps of $20 \mu \mathrm{m}$ ), whereas the piezo stage has a clearance of $100 \mu \mathrm{m}$ in the three directions with a sub-nm resolution. For each voxel, the fluorescence spectrum was measured with an integration time of $100 \mathrm{~ms}$.

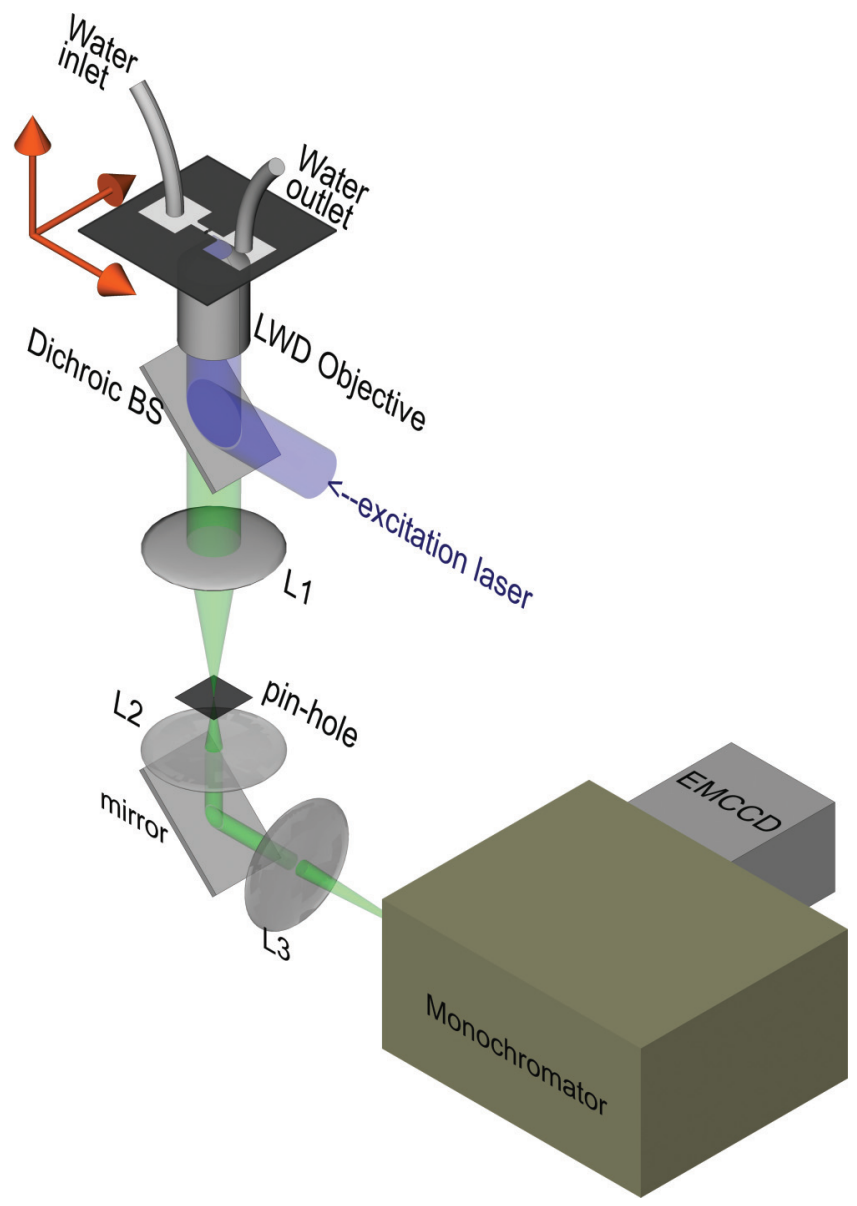

FIG. 2. (Color online) Schematic of the experimental setup. The microdiaphragm is fixed on a combined $X Y$ mechanical plate- $X Y Z$ piezo plate. The excitation light from the laser is focused inside the microsystem with a long working distance (LWD) objective. The luminescence is collected with the same objective, passes through a dichroic beamsplitter (dichroic BS), and is then focused on a pin hole with a lens (L1). The pin hole is imaged at the entrance of the monochromator with two lenses (L2 and L3). The monochromator and the EMCCD ensure the spectral analysis.

Performing thermal investigations inside eddies is the challenge of this work. A few alternative designs, devoted to the only hydrodynamic aspect of cavitation in microchannels, can be found in the literature $[16,17]$. We thus specifically developed a transparent micro channel device for optical purposes, based on the design presented in Refs. [3,4].

Precise description of the microfluid system is required since its dimensions are the input parameters for hydrodynamic modeling. The diaphragm under test was micromachined from a $H=124 \mu \mathrm{m}$-thick silicon foil that was anodically bonded onto a Pyrex substrate. A deep reactive ion etching process of a silicon wafer produced a well-controlled channel ( $W=500 \mu \mathrm{m}, L=3 \mathrm{~mm}$ ), interrupted at half-length by a rectangular diaphragm $(w=56 \mu \mathrm{m}, l=100 \mu \mathrm{m})$. A Pyrex cap with drilled apertures was then anodically bonded onto the channel. The final device is fully transparent and suitable for the optical setup. The structure of this Pyrex-silicon-Pyrex stacking device is closer to a silicon-Pyrex microchannel device $(H=126 \mu \mathrm{m}, W=500 \mu \mathrm{m}, w=60 \mu \mathrm{m})$ as formerly 


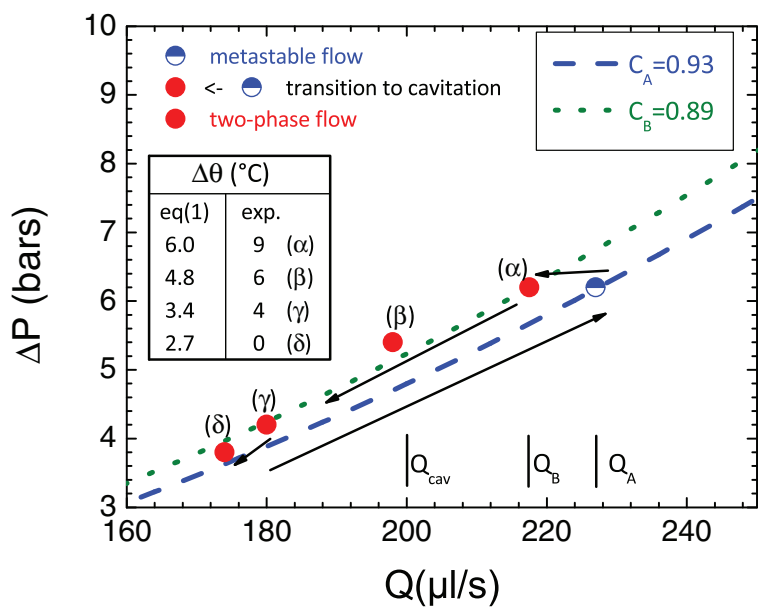

FIG. 3. (Color online) Relationship between the pressure drop $\Delta P$ and the flow rate $Q$ through the device under test. By increasing $\Delta P$, a metastable single liquid phase remains above $Q_{\text {cav }}$. The onset of cavitation goes with a drop of the flow rate from $Q_{A}$ to $Q_{B}(\alpha)$. By decreasing $\Delta P$, hydrodynamic cavitation remains $(\beta, \gamma)$ down to the desinence $(\delta)$. The dotted lines correspond to the theoretical relationship in single liquid and two phase flows. The inserted table indicates the maximum measured (exp) and calculated [Eq. (1)] increase of temperature for each cavitating flow regime.

characterized by our group (see Fig. 3(a) in Ref. [3]). We observed the relationship between the pressure drop $\Delta P$ across the device and the corresponding flow rate $Q$ (Fig. 3). It is known [3] that in a single liquid phase $\Delta P=\frac{\rho Q_{A}^{2}}{\left(2 C_{A}^{2} w^{2} H^{2}\right)}$, where $\rho$ is the liquid density and $C_{A}$ is a discharge coefficient. Above a critical flow rate $Q_{\text {cav }}$, the pressure downstream from the diaphragm falls below the saturate pressure and thus cavitation is likely to occur. Working with deionized water improves the occurrence of metastable states [3,4]. Nevertheless, at the end of cavitation onset, under a given pressure drop, the lowering of the flow rate obeys the relationship $\Delta P=\frac{\rho Q_{B}^{2}}{\left(2 C_{B}^{2} w^{2} H^{2}\right)}$, where $C_{B}$ $\left(\leqslant C_{A}\right)$ represents the discharge coefficient of the two-phase flow (Fig. 3). Furthermore, high speed optical observations demonstrated that bubbles formed by cavitation arise from very high shear rates downstream from the diaphragm at the border between the liquid jet and recirculating areas (see Fig. 11 in Ref. [4]).

In the microchannel under study, the expected values of $C_{A}$ and $C_{B}$ are 0.93 and 0.89 , respectively. The critical flow rate is $Q_{\text {cav }}=200 \mu \mathrm{L} / \mathrm{s}$, which corresponds to $\Delta P$ of 4.7 bars (for the detailed calculation see Ref. [3]). The onset of cavitation occurs when $Q=227 \mu \mathrm{L} / \mathrm{s}$ and $\Delta P=6.2$ bars (Fig. 3). Then, following the decreasing $\Delta P$ value, the two-phase cavitating flow disappears for $Q \leqslant 174 \mu \mathrm{L} / \mathrm{s}$ and $\Delta P=3.8$ bars. The fact that a two-phase flow remains even if $Q \leqslant Q_{c}$ has already been discussed in the literature [3]. Figure 4 illustrates the thermal map recorded in a plane located $10 \mu \mathrm{m}$ from the upper side of the channel. A comparison between an optical snapshot of the cavitating flow through the micro diaphragm with the temperature map enables a more precise understanding of the process. The most spectacular occurrence is the presence of a hot spot in areas where the vortices reach the highest intensity. The increase in temperature

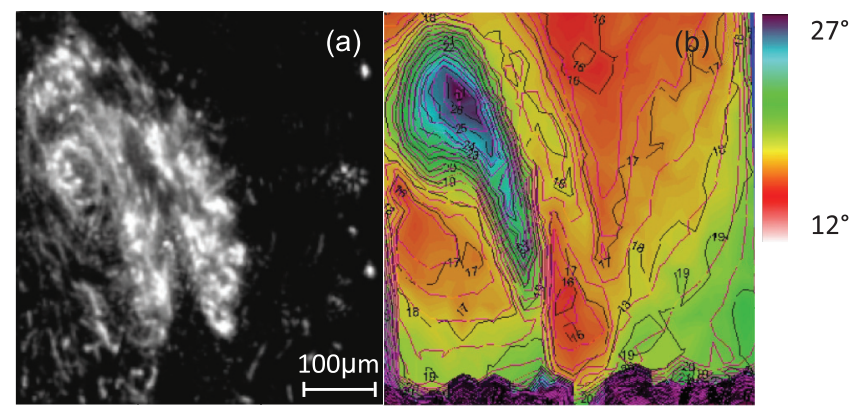

FIG. 4. (Color online) Comparison between (a) optical snapshot of a cavitating flow downside in the diaphragm. White areas represent the vapor phase. (b) Steady-state thermal reconstruction of data recorded under the same hydrodynamic conditions. The liquid jet emerging from the diaphragm flips towards the left side of the channel. Hot spots are located where the vortices display the strongest activity.

is around $10 \mathrm{~K}$ over a distance of $\sim 100 \mu \mathrm{m}$. It is unlikely that such an increase is the consequence of laminar viscous effects in the microchannels, because the expected level of these phenomena should be below $1 \mathrm{~K}$ [18]. Because of the necessity to analyze further the map temperature region, during another set of measurements, we focused the laser onto this area and recorded the temperature as a function of time starting from a single metastable liquid phase with $\Delta P=6.2$ bars and waiting till the onset of cavitation. That evolution is reported in Fig. 5. We can observe here that the temperature sharply increases in phase with the onset of cavitation. The thermal fluctuations display a stronger intensity in the cavitating regime than in the single liquid phase. The thermal gradient decreases together with the pressure drop [see data $(\alpha),(\beta),(\gamma)$ in Fig. 3] and disappears once cavitation switches off. We recorded similar maps to that in Fig. 4 at six different heights $(z)$ of the channel where the flow exhibits the strongest thermal gradient (fixing hydrodynamic condition of pressure $\Delta P=6.2$ bars). Figure 6(a) plots the thermal profiles along the $W$ axis (perpendicular to the length of the channel) for each $z$ value. These 3D investigations demonstrate that the thermal enhancement is located on the half upper side of the channel, specifically in the

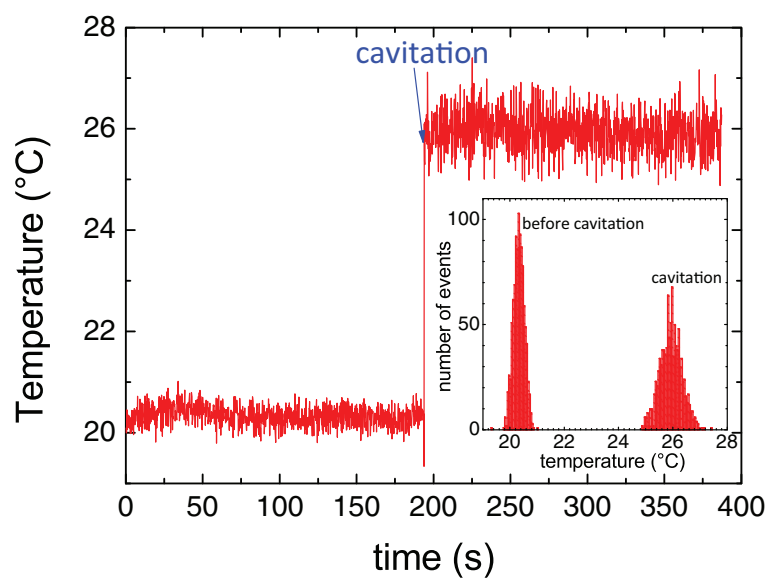

FIG. 5. (Color online) Time capture of the thermal signature of the cavitating flow regime. The thermal jump occurs simultaneously with the onset of cavitation. 


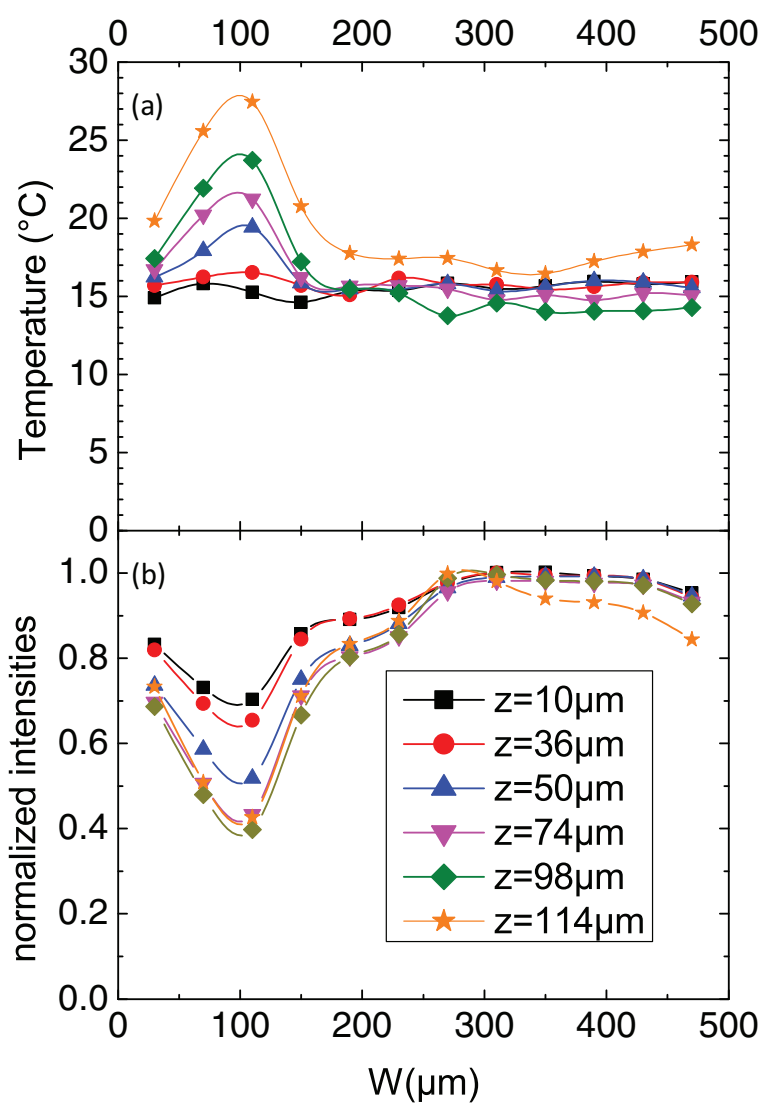

FIG. 6. (Color online) (a) Temperature profile recorded at different heights $z$ of the channel in the direction perpendicular to the flow, in the area where it exhibits the strongest thermal gradient. (b) Spatial variations of the thermally normalized intensity. Both data were recorded with a cavitating flow rate $Q=217 \mu \mathrm{L} / \mathrm{s}$.

recirculating area located between the liquid jet emerging from the diaphragm and the left side of the channel [see Fig. 4(a)]. The normalized intensity plotted in Fig. 6(b) is here only a function of the number of fluorescent emitting particles. So it may be regarded as information on the void fraction, because the nanoparticles are hydrophilic. The liquid phase reaches the highest temperature for $z=114 \mu \mathrm{m}$ and $W=100 \mu \mathrm{m}$ [Fig. 6(a)], where a maximum void ratio is found to be around $60 \%$ [Fig. 6(b)], so the thermal insulation is enhanced. That agrees with the physical model presented below. Note that for values of $W$ corresponding to the recirculating vortices located at the right side of the emerging jet $(150 \mu \mathrm{m}<W<$ $250 \mu \mathrm{m})$, the optical detection confirms the presence of a vapor phase but does not exhibit any noticeable increase of the temperature. That is a strong confirmation of the efficiency of the temperature detection, because it demonstrates that there is no correlation between the recorded emitted spectrum and the phase of the fluid under investigation.

Connexion between the temperature flow behavior and the hydrodynamic requires estimation of the power $\Pi$ dissipated by the cavitating regime. The transition from a single liquid phase toward a two-phase flow (that occurs under a fixed pressure drop $\triangle P$ ) occurs with a decrease of the flow rate from $Q_{A}$ to $Q_{B}$ and $\Pi=\Delta P\left(Q_{A}-Q_{B}\right)$ (Fig. 3). We assume that $\Pi$ is converted into heat by the viscous shear stress. As known, the thermal hot region is located in the zone where the larger flow structures break down into smaller ones, until kinetic energy is overwhelmed by the fluid's molecular viscosity. The power density of a thermal hot region of surface $S$ is defined as $p=\Pi /(S H)$; the snapshot in Fig. 4(a) allows us to consider that $S \cong(W / 4)^{2}$. Under conditions that (i) the liquid convection is not taken into account and (ii) the isothermal state is kept upside and downside of the volume under investigation, the resolution of the steady-state Fourier equation gives $\Delta \theta=p z(H-z) / 2 k$, where $0 \leqslant z \leqslant H$ is the vertical coordinate and $k=1 \mathrm{~W} / \mathrm{m} / K$ is the thermal conductivity of water. Since the maximum increase of temperature $\Delta \theta_{\max }$ occurs at $z=H / 2$, we obtain

$$
\Delta \theta_{\max }=\frac{1}{8} \frac{C_{A}-C_{B}}{C_{B}^{3}} \frac{\mu^{3}}{k \rho^{2}} \operatorname{Re}^{3} \frac{(w+H)^{3}}{W^{2} w^{2} H},
$$

where $\mathrm{Re}$ is the Reynolds number through the diaphragm. The thermal gap is enhanced when $W$ and the ratio $w / H$ tend to zero. Equation (1) results from a streamlined evaluation of two parallel isothermal planes separated by a distance $H$. We calculated and measured thermal gap values that are summarized in the inset of Fig. 3. We obtained $\Delta \theta_{\max }=6 \mathrm{~K}$ when $\Delta P=6.2$ bars, which reasonably accords with the recorded value in Fig. 4, but the Eq. (1) fails to describe that $\Delta \theta_{\max }=0$ once cavitation has disappeared. That is mainly due to the assumption of a constant $C_{A}-C_{B}$ value. However, as can be seen in Fig. 3, that last hypothesis remains reliable for the experimental cavitating data denoted $(\alpha),(\beta)$, and $(\gamma)$. Under these conditions, the maximum temperature gap recorded inside the core of dissipating eddies is not dissimilar to the one calculated from Eq. (1). Indeed, the temperature values calculated from Eq. (1) are slightly underestimated because the liquid phase fills only between $40 \%$ and $80 \%$ of the total volume $H S$. According to the previous equation, such a void ratio modifies the expected $\Delta \theta_{\max }$ to a value between $7.5 \mathrm{~K}$ and $15 \mathrm{~K}$, which corroborates the experimental results.

In conclusion, we measured a thermal signature of the transition from a single liquid phase toward a hydrodynamic cavitating flow in a microchannel. The temperature gradient matches with the onset of cavitation and with a drop of the flow rate. A physical model combining the corresponding hydrodynamic losses to the thermal effects has been proposed. That model furnishes orders of magnitude that are in a good agreement with the experimental results and that involve that the small size of the device enhances the thermal gradient. It is funded on the hypothesis that losses occur over a limited volume. However, heat transfers due to phase change may be considered because the overheating is located in the place where bubbles condensate. Further tests of different-sized microdiaphragms and of different-shaped designs, such as microventuris, are necessary, and they will contribute to obtaining a better understanding of these unexpected thermal effects.

This work was made possible through the French ANR LUNAPROBE Contract No. ANR 09-NANO-P057-36. The authors thank the NanOpTec center (Lyon, France) for access to the optical spectroscopy and the imaging confocal microscopy facilities. The authors are grateful to Dr. Mary B. Guillochon for careful proofreading of the manuscript. 
[1] R. Baviere, F. Ayela, S. Le Person, and M. Favre-Marinet, Phys. Fluids 17, 098105 (2005).

[2] J. Chevalier, O. Tillement, and F. Ayela, Phys. Rev. E 80, 051403 (2009).

[3] M. Medrano, P. J. Zermatten, C. Pellone, J. P. Franc, and F. Ayela, Phys. Fluids 23, 127103 (2011).

[4] M. Medrano, C. Pellone, P. J. Zermatten, and F. Ayela, Phys. Fluids 24, 047101 (2012).

[5] J. Sakakibara and R. Adrian, Exp. Fluids 26, 7 (1999).

[6] P. Lavieille, F. Lemoine, G. Lavergne, and M. Lebouche, Exp. Fluids 31, 45 (2001).

[7] M. Bruchhausen, F. Guillard, and F. Lemoine, Exp. Fluids 38, 123 (2005).

[8] P. Audebert, F. Bresson, R. Devillers, and G. Tribillon, Synthetic Met. 81, 315 (1996).

[9] S. Someya, D. Ochi, Y. Li, K. Tominaga, K. Ishii, and K. Okamoto, Appl. Phys. B 99, 325 (2010).
[10] V. K. Natrajan and K. T. Christensen, Meas. Sci. Technol. 20, 015401 (2009).

[11] M. Basson and T. S. Pottebaum, Exp. Fluids 53, 803 (2012).

[12] R. Segura, C. Cierpka, M. Rossi, S. Joseph, H. Bunjes, and C. J. Kähler, Microfluid. Nanofluid. 14, 445 (2013).

[13] D. Ross, M. Gaitan, and L. Locascio, Anal. Chem. 73, 4117 (2001).

[14] P. Chamarthy, S. V. Garimella, and S. T. Wereley, Int. J. Heat Mass Transf. 53, 3275 (2010).

[15] M. Martini, P. Perriat, M. Montagna, R. Pansu, C. Julien, O. Tillement, and S. Roux, J. Phys. Chem. C 113, 17669 (2009).

[16] C. Mishra and Y. Peles, Phys. Fluids 17, 013601 (2005).

[17] C. Mishra and Y. Peles, Phys. Fluids 18, 103603 (2006).

[18] G. Morini, Int. J. Heat Mass Transf. 48, 3637 (2005). 